\title{
THE CONTROL OF COMMUNICABLE DISEASES IN PSYCHIATRIC HOSPITALS.*
}

By E. B. SAYE, M. D.,

Pathologist, Harrisburg State Hospital, Harrisburg, Pa.

Although the practical control of communicable diseases in state hospitals may require certain morifications of methods employed elsewhere, the principles upon which all hygienic measures depend are universal.

I shall, therefore, present what seems to be a consensus of modern opinion regarding the essential epidemiological features of these diseases, and then attempt to show how these facts apply to conditions in hospitals for mental diseases.

Fifty years ago che germ theory of disease had not been accepted, and a belief in the doctrine of spontaneous generation still lingered in the minds of some medical men. The origin of communicable diseases was then ascribed to noxious emanations from the bodies of sick persons or to the pernicious influence of air charged with miasm.

To-day, however, we are convinced that all communicable diseases are directly attributable to animal or vegetable parasites; that these diseases spread only as their causal agents are transferred from the sick to the well; and that only by their secretions and excretions do infected individuals endanger others.

Under the term communicabl. diseases are included: all of the acute eruptive fevers, as smallpox, scarlet fever, and measles; the respiratory infections, for example, influenza and tuberculosis; such enteric affections as dysentery, typhoid, and uncinariasis; certain protozoan infections whose transmission depends upon the bite of an insect, for instance, malaria: a few diseases whose principal manifestation is a skin lesion, for example, favus and

* Read before the Thirty-Seventh Semi-Annual Meeting of the Association of Trustees and Medical Superintendents of the State and Incorporated Hospitals for the Insane and Feeble Minded of Pennsylvania, Harrisburg, May 6, I92I. 
scabies; the venereal diseases; and, from the standpoint of the hygienist, those diseases also, the exact nature of whose communicability is not yet determined, but which are apparently infectious, notably encephalitis lethargica.

The agencies through which it is possible for communicable diseases to spread are: contact, animal or human carriers, water, and milk or other food.

It was formerly believed that most of the communicable diseases were air-borne. Merely entering a sick-room was then regarded as an instance of exposure by direct contact. We now know that air plays an exceedingly small part in the transmission of disease. Park ${ }^{1}$ states that there are few authenticated instances of the aërial transmission of infection beyond a patient's room, and Marshall ${ }^{2}$ says that, even in crowded rooms, the greater probability is that infection comes about through actual contact.

The term direct contact now refers to the immediate transfer of infectious material from one to another. The transfer of some of the respiratory infections may take place directly, as, for example, when a diphtheritic patient coughs into the face of an attendant. The venereal diseases are usually transmitted by absolute contact. In general, however, the transfer of infection is more indirect.

Fomites, once considered an important source of infection, are now disregarded in most diseases. The term indirect contact now refers almost entirely to the transfer of infection by objects freshly contaminated by infectious excretions. As Park" puts it, "the probability of such transfer is directly proportionate to the time elapsing since contamination of the "fomes." "

The majority of infections are acquired either by inhalation or by ingestion. Moreover, infectious material is conveyed to the mouth or nose more frequently by the hand than in any other way. The hands of an attendant may become soiled by the discharges of a patient. His fingers then touch some object, as a fork or spoon, which goes to his own mouth or to the mouth of another. Such commonly-used objects as doorknobs, handkerchiefs, towels, and drinking cups may likewise facilitate the spread of infection. Undoubtedly, the most important factor in the distribution of communicable disease is the hand, and it has become increasingly apparent that the hand-to-mouth route of infection is the usual one." 
The contamination of milk and other food results almost entirely from infected hands, and is, therefore, but another instance of contact-infection; while a contaminated water supply simply represents on a large scale the transfer of human excreta to human mouths.

Besides the diseased individual, there is another important source of infection, namely, the carrier. By the term carrier is meant a person who, without symptoms of a communicable disease, harbors and disseminates the specific microörganisms." A carrier state may occur during convalescence, or it may continue following recovery from an iniectious disease; or, following exposure to infection, a healthy person may become a carrier.

Some of the lower animals may also communicate disease to man, either mechanically or by serving as intermediate hosts for the causal agent. However, the most important distributor of human sickness is man himself.

In the foregoing discussion, we have dealt briefly with the ways in which communicable diseases spread. We shall now consider the methods of preventing the spread of these diseases. The means of control are: recognition, isolation, disinfection, and immunization.

I cannot emphasize too strongly the importance of early diagnosis in the prevention of infectious diseases. Measles, for instance, is highly communicable during the pre-eruptive stage.

The case with mild symptoms also deserves serious consideration. A number of serious respiratory affections, for instance, begin with symptoms resembling those of an ordinary cold. So eminent an authority as Park ${ }^{\circ}$ says that there is no laboratory method of differentiating between a cold and influenza. And Foster' has recently shown that bronchopneumonia frequently passes under a diagnosis of bronchitis. Furthermore, serious secondary infections are readily implanted upon tissues rendered vulnerable by slight inflammations. Again, mild cases of diarrhea may be due to typhoid or dysentery bacilli. Bates,' of the Panama Canal Zone Health Department, reported a bacteriologically-controlled series of mild, atypical typhoid cases, and remarked their importance as sources of endemic infection.

Not only must we recognize the early case and the case with mild or atypical symptoms, but we must also have in mind the carrier. 
The appearance on our wards of such diseases as typhoid, diphtheria, or meningitis, suggests a carrier, and indicates a bacteriologic search for such person among the patient's associates.

If we would prevent epidemics, we must isolate every case of communicable disease during the period of infectivity. The period of communicability continues as long as definite lesions persist, and until microörganisms, known to have a definite etiologic relation to the disease concerned, disappear from the excretions. Some diseases are quite infectious during the prodromal period. Hence, during an epidemic, we must isolate not only the actual cases, but also all contacts who show an elevation of temperature.

There are two systems of disinfection: concurrent and terminal. The term concurrent applies to the disinfection, while the disease is in progress, of all discharges and of all articles which come in contact with the patient; terminal refers to the disinfection of the premises when the patient has vacated them.

Terminal disinfection has practically been abandoned. Fumigation methods, as ordinarily carried out, are notoriously uncertain in action. Furthermore, they are entirely unnecessary if concurrent disinfection has been faithfully employed. The most satisfactory method of terminal disinfection is to sun and air the sick-room thoroughly, after carefully scrubbing its floors and walls.

At the Ellis Island Hospital, contagious cases constitute seventy per cent of the admissions. The patients are all served by the same laundry and kitchen, and all handled by the same personnel. They have discontinued terminal disinfection in this institution, and rely entirely on concurrent disinfection and simply scrubbing and airing the vacated rooms. Yet only two per cent of their patients develop cross-infections.

Regarding the use of vaccines and anti-serums for immunizing purposes, there are very few diseases, indeed, in which these agents are of proved value. Smallpox, rabies, tetanus, typhoid and paratyphoid, and to a lesser extent, diphtheria, cholera, and bacillary dysentery are probably the only ones.

We have now considered the principles underlying the spread and control of communicable diseases. We have seen that the patient's discharges contain the source of danger; that human contact is the most important factor in spreading infection; and 
that the route of conveyance is most frequently from hand to mouth. We have noted also the great importance of the early case, the mild case, the unrecognized case, and the carrier, in the spread of infection. Likewise we have noted the necessity for isolation and concurrent disinfection. With these fundamental facts in mind, let us consider their specific relation to the problems of the state hospital.

Although a few suggestions will be made regarding the construction of contagious hospitals, the technic of medical asepsis, the use of disinfectants, and personal hygiene, it is not my intention to treat these subjects comprehensively. ${ }^{10}$ Nor are the methods, outlined for the control of communicable diseases in general, sufficient for the management of every communicable malady. Malaria, for instance, is not affected by measures designed to prevent bacterial diseases.

In psychiatric hospitals, communicable diseases are transmitted almost entirely by contact-infection. Food, water, and carriers are relatively less active sources of infection.

Every hospital should secure as pure a water supply as it is possible to obtain. However, water is not a frequent source of disease in institutions. Typhoid is the principal disease transmitted by water. Water-borne epidemics do occur, and, when they arise, affect almost simultaneously a large number of persons. Yet there is no doubt that, in the past, many typhoid epidemics have been erroneously imputed to bad water, whereas more immediate sources of infection, such as contact, carriers, or mild cases, were not carefully excluded.

Milk, too, is a minor factor in the spread of disease in institutions. With the rare exception of bovine tuberculosis, the diseases transmitted by milk are all due to the insanitary handling of the milk. I would counsel vigilance in the supervision of all foodhandlers. Every infected person, carrier, or convalescent should be carefully excluded from such duty.

The human carrier is undoubtedly responsible for some epidemic disease in state hospitals. However, the carrier distributes disease in the same way as does the sick person; it is only by contact with the infectious discharges of the carrier that we are endangered.

The conditions in all psychiatric hospitals are such as to favor the dissemination of diseases that may be spread by contact- 
infection. The patients spend the greater part of their time, closely associated with other patients, on a ward. Nor is the ward itself an isolated unit. In the kitchen, dining-room, amusement hall, sewing room, and elsewhere, the patients mingle with those from other wards. Moreover, visitors and attendants introduce into the wards infections which they have acquired outside the hospital. Again, it is necessary to utilize the help of patients in the ward work. Frequently, the same patient will, at one time, assist in performing toilet service for some unclean patient, and, at another time, help serve meals. Add to the foregoing statements the fact that we cannot improve the insanitary personal habits of some of our patients, and you have a fair account of the possibilities of contact-infection in hospitals for mental cases.

To prevent communicable diseases, it is not necessary to restrict the mingling of our patients nor to modify materially the normal routine of hospital life. It is necessary to detect and isolate promptly all infectious cases occurring on our wards.

Every state hospital should have special wards for contagious cases. The experience of the Ellis Island Hospital,' and that of Richardson " at the Providence, R. I., City Hospital have demonstrated the possibility of handling contagions diseases successfully even on the wards of a general hospital. However, it is certainly unwise to keep infectious cases on the general wards of a psychiatric hospital.

The contagious wards may safely be situated near the other hospital buildings. The construction of the wards may be similar to that of the general wards, the single rooms being used for individual contagious cases, and the day rooms for convalescents, or, perhaps, for suspicious contacts. Stokes," of the Dermatologic Department of the Mayo Clinic, who has studied exhaustively the question of contagious hospital construction, advocates the single-room type of building. Ample facilities for sterilization should be provided.

A more important matter than the architecture of the contagious wards, is the rigid observance of a technic adequate to prevent cross-infections within the hospital. Every object removed from a sickroom must be regarded as infected until sterilized. No unsterile article may be taken from the room of one patient, or class of patients, to that of another. A separate gown, placed 
near the entrance to each room, is put on when entering and removed upon leaving. The hands must invariably be disinfected upon leaving a room.

A two per cent emulsion of creolin can be recommended as an efficient, practical disinfectant for the hands." This emulsion is also useful for such purposes as cleaning bedsteads or washing out bathtubs. Allowed to act for one hour, it may be used to disinfect clothing, utensils taken from isolation rooms, and discharges. For the latter purpose, thorough incorporation of the excreta with the disinfectant is important. The disinfection of most of the articles used on contagious wards may also be accomplished by boiling, which is a much more certain method. Gowns and other linen may be sterilized by steam under pressure.

The attendants on the general wards are incompetent to nurse contagious cases. They lack training. Indeed, many of them do not expect to continue in hospital work, and, therefore do not care to learn how to nurse the sick. For the personnel of our contagious wards we must select a few reliable attendants, and teach them the principles of infection and of medical asepsis.

If we isolate, as we should, every infectious case, our contagious ward will seldom be empty. And, to be effective, isolation must be thorough. Neglect to isolate an occasional infectious case may mean the failure of our whole system of prophylaxis.

More urgent, perhaps, than the need for a contagious hospital, is that of providing pavilions for the tuberculous patients. The experience of the Georgia State Sanitarium ${ }^{12}$ sufficiently emphasizes the value of such pavilions, not only as a means of segregating the tuberculous patients, but as an efficient therapeutic measure as well. Following the construction of pavilions at the Georgia institution, the mortality rate for tuberculosis fell, within three years, from 28 to $7 \frac{1}{2}$ per cent.

Since the prevention of communicable disease is so largely a personal matter, a few elementary hygienic facts ought to be universally known. As physicians, we should, whenever and whereever possible, aid in the diffusion of this knowledge. We should impress upon all of our attendants, and upon docile patients, such facts as the importance of keeping the fingers away from the mouth and nose, and of washing the hands before eating.

Reviewing, then, the question of a communicable disease control in psychiatric hospitals, it is obvious that a clear conception of 
the epidemiology of these diseases is essential. In addition to this, the early and thorough isolation of infected patients, a properly trained personnel to nurse the infectious cases, and the encouragement of better personal hygiene, are the considerations which seem to be of most importance.

\section{REFERENCES.}

I. Park, W. H.: Public Health and Hygiene, Philadelphia, Lea \& Febiger, 1920, p. 218.

2. Marshall, C. E.: Microbiology, Ed. 2, Philadelphia, P. Blakiston's Son \& Co., 1917, p. 665.

3. Park, W. H. and Williams, A.: Pathogenic Microorganisms, Ed. 7, Philadelphia, Lea \& Febiger, 1920, p. 166.

4. Hand-to-Mouth Infection, editorial, New York M. J., IIo: 332 (Aug. 23), 1919.

5. The Control of Communicable Diseases, Miscellaneous Publication 24, U. S. P. H. S., 1920, p. 3.

6. Park, W. H.: Public Health and Hygiene, Philadelphia, Lea \& Febiger, 1920, p. 75.

7. Foster, N. B.: Notes on the Diagnosis of Bronchopneumonia and its Complications, Am. J. M. Sc., I6I : I (Jan.) I921.

8. Bates, L. B., J. A. M. A., 50: 585 (1908) quoted by Chapin, C. V.: Sources and Modes of Infection, Ed. 2, New York, John Wiley \& Sons, 1916, p. 57.

9. Wilson, J. G.: Infectious Diseases of Children, Bulletin 95, U. S. P. H. S., (Oct.) 1918.

10. In addition to references already given, the following articles by $D . L$. Richardson will be of interest:

Medical Asepsis in French and English Hospitals, Mod. Hosp., 12: 330 (May) 1919.

Aseptic Nursing in American Hospitals, Mod. Hosp., 13: 1o (Aug.) Igrg.

Management of Contagious Disease Hospitals, Mod. Hosp., 13: 190 and 13: 468 (Sept. and Dec.) I9I9.

The Prevention of Cross-infections in the Children's Ward of a General Hospital, Mod. Hosp., 14: 36I (May) 1920.

Hospital and Home Isolation of Infectious Diseases: Their Relative Values, Mod. Hosp., 14: 99 (Feb.) 1920.

II. Richardson, D. L.: Management of Contagious Disease Hospitals, Mod. Hosp., 13: 379 (Nov.) 1919.

I2. Stokes, J. H.: The Organization and Methods of Contagious Disease Services, Penn. M. J., 22: 729 (Aug.) 1919.

13. Green, E. M.: Personal communication. 